\title{
UBER DIE BEDEUTUNG DES MAXIMALERGEBNISSES BEI BEURTEILUNG DER ZUGKRAFT DES PFERDES
}

\author{
Mikko Varo und Viljo Vainikainen \\ Zentrale für Landwirtschaftliche Forschung, Abteilung für Haustierzüchtung, Tikkurila
}

Eingegangen am 2. V. 1958

Seitdem man im Jahre 1936 den an einem Kraftwagen befestigten Zugwiderstandsmesser in Gebrauch genommen hatte, um die Zugkraft von Hengsten zu beurteilen, galt es als Zielsetzung, die grösstmögliche Zugkraft zu messen. Die Begründung war, dass nur so die nach Zugkraft und Zugsicherheit sowie Charakterstärke wertvollsten Spitzentiere, durch die die Zugfähigkeit der Rasse am sichersten weiterzuentwickeln sei, herausgefunden werden könnten. Da die Zugfähigkeit des Pferdes eine Eigenschaft ist, die nur bei gemeinsamen Versuchsgelegenheiten, bei Vorführungen für die Stammbucheintragung, vergleichbar geprüft werden kann und da wegen der geringen Zahl der Pferde keine Möglichkeit besteht, innerhalb der Betriebe unter den einzelnen Tieren einen Vergleich anzustellen, scheint das Messen der grösstmöglichen Zugkraft das einzige für die Praxis geeignete Verfahren zu sein, ein grundlegendes Material für die auf die erbliche Zugkraft der Individuen bezogene Auslese zu gewinnen. Danach hat man mit dem benutzten Versuchsverfahren gestrebt, bei dem ein in bezug auf das Gewicht des Tieres relativer Zugwiderstand nach einem stufenweise gezogenen Abschnitt oder einer 50 Meter messenden Zugstrecke hinzugefügt wird. Der Zugwiderstand, der bei der ersten Stufe bei einem vierjährigen Pferd $25 \%$ des Lebendgewichtes ausmacht, steigt nach jeder Stufe um fünf Prozent des Lebendgewichtes, und als endgültiges Zugergebnis wird die Ordnungszahl der letzten einwandfrei gezogenen Stufe, die Stufenzahl, vermerkt. In erster Linie wurde auf Forderung der Trabrennsport betreibenden Pferdezüchter in der Vorschrift jedoch im Jahre 1952 eine Änderung vorgenommen, die ein Absehen von dem besprochenen Maximalprinzip im Gefolge hatte. Damals nämlich wurde beschlossen, dass die höchste im Zugversuch zu erlangende Punktmenge die durch die achten Stufe gewonnenen acht Punkte seien. Dies bedeutete natürlich zugleich, dass es nicht mehr notwendig sei, den Zugversuch bis zur grösstmöglichen Stufenzahl fortzusetzen, sondern der Zugversuch konnte nach der achten Stufe beendet werden. Die besagte Änderung bewirkte denn auch einen deutlichen Rückgang in den durchschnittlichen Stufenzahlen, wie Tabelle 1 erkennen lässt. Die Abnahme der Stufen- 
zahlen scheint zwar später haltgemacht zu haben, aber in Anbetracht der starken Verminderung in der Anzahl der für das Stammbuch angebotenen Hengste scheint es offensichtlich, dass das Interesse, das höchste Zugergebnis zu erlangen, infolge der Änderung der besprochenen Vorschrift merklich abgenommen hat.

Mit Rücksicht auf das Weiterentwickeln des Zugversuchs wäre es notwendig, zu ermitteln, ob das Maximalprinzip bei Beurteilung der Zugkraft der Pferde von Bedeutung ist oder ob man davon ausgehen kann, wie heute, dass das Ziehen von acht Stufen schon eine so gute Zugkraft beweist, dass sie unter den gegenwärtigen Verhältnissen als für alle Pferde ausreichend angesehen werden kann. Wie aber aus Tabelle $1 \mathrm{zu}$ ersehen, entspricht das Ergebnis der achten Stufe bei weitem nicht dem Mittelwert der für das Stammbuch genehmigten Hengste, und in den besten Jahren, als das Interesse für den Zugversuch noch allgemein positiv gewesen ist, hat auch der Mittelwert aller geprüften Hengste die Grenze der achten Stufe deutlich überschritten.

Um darzulegen, welche Bedeutung den bestmöglichen und den schwächeren Zugresultaten als Indikatoren erblicher Zugkraft zukommt, wird im folgenden die Heritabilität des Zugerfolges auf Grund der besten und der schlechtesten Zugresultate von wenigstens zweimal geprüften Hengsten beurteilt. Der grösste Teil der 489 Hengste des Materials hat zweimal am Versuch teilgenommen. Die Untersuchung ist so vorgegangen, dass der Anteil der zwischen den Vätern bestehenden Varianz an der Gesamtvarianz der prozentualen Zugergebnisse der Söhne sowohl nach den

Tabelle 1. Die Entwicklung der Stufenzahlen in den Jahren $1940-57$ bei vierjährigen Hengsten.

\begin{tabular}{|c|c|c|c|c|c|c|}
\hline \multirow[t]{3}{*}{ Jahr } & \multicolumn{3}{|c|}{ Mittelwerte der Stufenzahlen } & \multicolumn{3}{|c|}{ Anzahl der Hengste } \\
\hline & \multicolumn{2}{|c|}{ Angenommen } & \multirow[t]{2}{*}{ Abgelehnt } & \multicolumn{2}{|c|}{ Angenommen } & \multirow[t]{2}{*}{ Abgelehnt } \\
\hline & Arbeitspferde & $\begin{array}{c}\text { Universal- } \\
\text { pferde }\end{array}$ & & Arbeitspferde & $\begin{array}{c}\text { Universal- } \\
\text { pferde }\end{array}$ & \\
\hline 1940 & 8.14 & 8.44 & 6.77 & 58 & 9 & 44 \\
\hline 1941 & 7.96 & 7.60 & 6.63 & 67 & 5 & 64 \\
\hline 1942 & 8.38 & 8.36 & 5.39 & 53 & 11 & 31 \\
\hline 1943 & 8.03 & 8.58 & 6.19 & 62 & 1 & 75 \\
\hline 1944 & 8.31 & 8.73 & 7.08 & 84 & 11 & 84 \\
\hline 1945 & 8.60 & 8.50 & 7.25 & 70 & 12 & 48 \\
\hline 1946 & 8.83 & 9.56 & 8.01 & 120 & 16 & 91 \\
\hline 1947 & 9.24 & 9.85 & 7.89 & 167 & 13 & 200 \\
\hline 1948 & 8.96 & 9.44 & 7.99 & 165 & 9 & 199 \\
\hline 1949 & 8.90 & 9.27 & 7.51 & 92 & 11 & 141 \\
\hline 1950 & 8.78 & 8.78 & 7.54 & 60 & 9 & 124 \\
\hline 1951 & 9.50 & 8.86 & 7.90 & 42 & 7 & 53 \\
\hline 1952 & 7.87 & 8.40 & 6.02 & 46 & 5 & 59 \\
\hline 1953 & 8.41 & 8.20 & 7.06 & 44 & 5 & 30 \\
\hline 1954 & 9.07 & - & 8.31 & 29 & - & 13 \\
\hline 1955 & 8.64 & 9.50 & 8.27 & 47 & 2 & 11 \\
\hline 1956 & 8.79 & 8.60 & 8.00 & 48 & 5 & 24 \\
\hline 1957 & 8.95 & 8.60 & 8.00 & 37 & 5 & 13 \\
\hline
\end{tabular}


Tabelle 2. Die Abhängigkeit der Beurteilungssicherheit der Zugkraft von der Grösse des Ergebnisses.

\begin{tabular}{|c|c|c|c|c|c|}
\hline \multirow{2}{*}{$\begin{array}{l}\text { Zugergebnisse, } \\
\text { mit denen die } \\
\text { Berechnungen } \\
\text { angestellt } \\
\text { worden sind }\end{array}$} & \multirow{2}{*}{$\begin{array}{l}\text { Anteil der } \\
\text { zwischen den } \\
\text { Vätern beste- } \\
\text { henden Va- } \\
\left.\text { rianz }{ }^{1}\right) \\
\%\end{array}$} & \multirow{2}{*}{$\begin{array}{l}\text { Genaunig- } \\
\text { keit der } \\
\text { Werte der } \\
\text { Söhne } \\
\%\end{array}$} & \multicolumn{2}{|c|}{$\begin{array}{l}\text { Mittelwerte der } \\
\text { Zugleistungen }\end{array}$} & \multirow{2}{*}{$\begin{array}{c}\text { Streuung } \\
\text { innerhalb } \\
\text { der Väter } \\
\\
\%\end{array}$} \\
\hline & & & $\begin{array}{c}\text { prozentualer } \\
\text { Zugwi- } \\
\text { derstand }\end{array}$ & $\begin{array}{l}\text { Anzahl } \\
\text { der } \\
\text { Stufen }\end{array}$ & \\
\hline die besten $\quad \ldots \ldots \ldots$ & $11.19(1)$ & 45 & 66.8 & 9.36 & 6.872 \\
\hline die schwächsten .... & $1.34(-)$ & 5 & 54.5 & 6.90 & 12.091 \\
\hline Mittelwerte für alle & $12.95(2)$ & 52 & 61.0 & 8.20 & 7.930 \\
\hline
\end{tabular}

besten als nach den schlechtesten Zugergebnissen der Sohnhengste berechnet worden ist. Die so erhaltenen Zahlen sind aus Tabelle 2 zu ersehen. Das Material umfasst 133 Väter.

Die statistische Zuverlässigkeit der auch für die besten Zugergebnisse erhaltenen innerhalb der Klasse bestehenden Korrelation 0.11 ist zwar nur 95prozentig, aber das gewonnene Ergebnis weist jedenfalls recht deutlich darauf hin, dass die Bedeutung des besten Zugergebnisses als Indikator erblicher Neigung deutlich grösser ist als die der schlechtesten. Da die innerhalb der Klasse bestehende Korrelation 0.11 sich auf die zwischen den Söhnen herrschende genetișche Korrelation 0.25 gründet, beweist der vierfache Wert der besagten Korrelation, 0.45, die Genauigkeit der besten Zugergebnisse der Söhne als Indikator erblicher Zugneigung. Die Zahl 0.45 ist zugleich eine Bewertung der Heritabilität der in unserem Zugversuch gemessenen Zugkraft, wenn es sich um die besten Zugergebnisse der Hengste handelt. Sie is beträchtlich hoch gegenüber dem von den Verfassern in der vorhergehenden Untersuchung (2) erhaltenen durchschnittlichen Wert 0.17. Aber die früher (1) berechnete zwischen Vätern und Söhnen bestehende Korrelation $r=0.33 \pm 0.11$, berechnet nach Ergebnissen von wenigstens sechs Stufen und nach einem Material, in dem die Mütter ausschliesslich Resultate von sechs Punkten oder der Zugkraft nach das bestmögliche Stutenmaterial vertraten, weist auch darauf hin, dass die besten Zugerfolge als Indikatoren erblicher Zugkraft von grösserer Bedeutung als die schlechteren sind. Die für alle Zugerfolge und für ein Material von Müttern aller Stufen berechnete entsprechende Korrelation machte nämlich nur $0.17 \pm 0.10$ aus. Auf Grund des nun erhaltenen Ergebnisses kann man denn auch zu der Auffassung gelangen, dass ein wichtiges Mittel zur Steigerung der Zuverlässigkeit des Zugversuchs es wäre, in diesen wieder das anfangs bei ihm in Kraft gewesene Prinzip der Höchstleistung zurückzuführen, das darauf abgesehen ist, die grösstmögliche Zugkraft der zu prüfenden Pferde zu messen. Gewiss ist zugleich auch weiterhin danach zu streben, dass die zu den Versuchen gebrachten Pferde möglichst gleichmässig und möglichst gut eingearbeitet sind, damit ein Misslingen des Versuches selbst nicht vorkäme. 
Die Verfasser schlugen in ihrer früheren Untersuchung vor, dass als endgültiger Zugerfolg mehrmals geprüfter Pferde der Mittelwert aller Zugleistungen eingetragen würde. Diesen Vorschlag begründeten sie damit, dass diejenigen Pferde, die bei ihrem Versuch einmal völlig versagt hatten, auch bei ihren übrigen Versuchen durchschnittlich schlechtere Ergebnisse erwiesen als diejenigen, die nur einen gelungenen Versuch vorgeführt hatten, so dass das Misslingen der Prüfung auch in erblichen Faktoren begründet gewesen sein mag. In Tabelle 2 erweist denn auch die dritte Reihe, die aus den Mittelwerten aller Zugerfolge der Söhne berechnet worden ist, dass der Vorschlag berechtigt gewesen ist. Der Mittelwert aller Versuchsergebnisse gibt eben ein noch etwas sichereres Bild von der erblichen Zugkraft der Pferde als die einzelnen besten Zugleistungen.

$$
\text { Schlus sfolgerungen }
$$

Bei einem Material von 489 wenigstens zweimal im Zugversuch gewesenen Hengsten wurde festgestellt, dass der Anteil der zwischen den Vätern bestehenden Varianz an der Gesamtvarianz der Söhne bei Berechnung nach den besten Zugleistungen der Söhne $11.19 \%$ und bei Berechnung nach den schwächsten derselben Söhne 1.34 \% ausmachte. Wurden die Berechnungen nach den Mittelwerten aller Zugergebnisse der Söhne angestellt, so belief sich der Anteil der zwischen den Vätern bestehenden Varianz auf $12.96 \%$. Auf Grund der Ergebnisse wird vorgeschlagen, den Zugversuch als Maximalversuch wiederherzustellen, durch den die grösstmögliche Zugkraf des Pferdes gemessen werden kann.

\section{LITERAT UR VERZEICHNIS:}

(1) VARO, M. 1947. Suomalaisen hevosen vetovoiman kehittämisestä. Summary: The development of the finnish Horses' pulling power. Maatal.tiet. aikak. 19: 69-82.

(2) VARo, M. \& VAINIKAINEN, V. 1958. Uber den für den ungleichgewichtigen und ungleichaltrigen Pferde aufzustellende Zugwiderstand. Selostus: Eripainoisille ja eri-ikäisille hevosille asetettava vetovastus. Ibid.

S E L OS T U S :

MAKSIMITULOKSEN MERKITYKSESTÄ HEVOSEN VETOVOIMAN ARVOSTELUSSA

MikKo Varo ja Viljo VAINiKaINen

Maatalouden tutkimuskeskus, kotieläinjalostuslaitos, Tikkurila

Vähintään kahdesti vetokokeessa olleen 489 oriin aineistolla todettiin, että isien välisen muuntelun osuus poikien kokonaismuuntelusta oli poikien parhaista vetotuloksista laskien $11.19 \%$ ja samojen poikien heikoimmista vetotuloksista laskien $1.34 \%$. Kun laskelmat suoritettiin poikien kaikkien vetotulosten keskiarvoista, oli isien välisen muuntelun osuus $12.96 \%$. Tuloksen perusteella ehdotetaan vetokoe palautettavaksi maksimikokeeksi, jolla hevosen suurin mahdollinen vetovoima on mitattavissa. 